\title{
The effect of healthy dietary consumption on executive cognitive functioning in children and adolescents: a systematic review
}

\author{
J. F. W. Cohen ${ }^{1,2 *}$, M. T. Gorski ${ }^{3}$, S. A. Gruber ${ }^{4,5}$, L. B. F. Kurdziel ${ }^{6}$ and E. B. Rimm ${ }^{2,7,8}$ \\ ${ }^{1}$ Department of Health Sciences, Merrimack College, North Andover, MA 01845, USA \\ ${ }^{2}$ Department of Nutrition, Harvard T.H. Chan School of Public Health, Boston, MA 02115, USA \\ ${ }^{3}$ Department of Health Policy, Harvard Graduate School of Arts and Sciences, Harvard University, Cambridge, MA O2138, USA \\ ${ }^{4}$ Cognitive and Clinical Neuroimaging Core, McLean Hospital, Belmont, MA 02478, USA \\ ${ }^{5}$ Department of Psychiatry, Harvard Medical School, Boston, MA 02115, USA \\ ${ }^{6}$ Department of Psychology, Merrimack College, North Andover, MA 01845, USA \\ ${ }^{7}$ Department of Epidemiology, Harvard T.H. Chan School of Public Health, Boston, MA 02115, USA \\ ${ }^{8}$ Department of Medicine, Channing Division of Network Medicine, Brigham and Women's Hospital and Harvard Medical \\ School, Boston, MA 02115, USA
}

(Submitted 21 January 2016 - Final revision received 27 June 2016 - Accepted 1 July 2016 - First published online 4 August 2016)

\begin{abstract}
A systematic review was conducted to evaluate whether healthier dietary consumption among children and adolescents impacts executive functioning. PubMed, Education Resources Information Center, PsychINFO and Thomson Reuters' Web of Science databases were searched, and studies of executive functioning among children or adolescents aged 6-18 years, which examined food quality, macronutrients and/or foods, were included. Study quality was also assessed. In all, twenty-one studies met inclusion criteria. Among the twelve studies examining food quality $(n 9)$ or macronutrient intakes $(n$ 4), studies examining longer-term diet $(n 6)$ showed positive associations between healthier overall diet quality and executive functioning, whereas the studies examining the acute impact of diet ( $n 6$ ) were inconsistent but suggestive of improvements in executive functioning with better food quality. Among the ten studies examining foods, overall, there was a positive association between healthier foods (e.g. whole grains, fish, fruits and/or vegetables) and executive function, whereas less-healthy snack foods, sugar-sweetened beverages and red/processed meats were inversely associated with executive functioning. Taken together, evidence suggests a positive association between healthy dietary consumption and executive functioning. Additional studies examining the effects of healthier food consumption, as well as macronutrients, on executive functioning are warranted. These studies should ideally be conducted in controlled environments and use validated cognitive tests.
\end{abstract}

Key words: Executive functioning: Systematic reviews: Child and adolescent diet: Healthy dietary patterns

Cognitive functioning plays a crucial role in both academic achievement $^{(1,2)}$ and health-related decision making ${ }^{(3-5)}$ in children and adolescents. Executive functioning (EF) is a particularly important domain within cognitive processing and consists of the mental capacity to make goal-directed behaviours, including inhibitory control, working memory, attention and planning. EF skills develop throughout childhood and adolescence and parallel the developmental changes that occur in the brain throughout this period ${ }^{(6)}$.

Diet is likely to play an important role in cognitive functioning and $\mathrm{EF}$, and previous literature reviews have focused on the impact of breakfast consumption and its association with general child cognition and/or academic performance. A review of forty-one publications by Hoyland $e t a l .{ }^{(7)}$ concluded that compared with skipping breakfast, children who consumed breakfast demonstrated improved cognitive performance based on various measures of cognitive functioning and academic achievement. The observed effect was greater among nutritionally at-risk or stunted children compared with those who were well nourished. However, the quality of the breakfast was not assessed given the paucity of publications in that area, and the breakfasts cited within the studies reviewed were typically of poor nutritional quality (e.g. high in refined carbohydrates and sugar). More recently, studies have directly examined food quality and cognitive functioning. A systematic review of this topic will help inform school feeding programmes, wellness policies and paediatric feeding guidelines given the potential importance of diet on academic performance.

Abbreviations: CPM, Coloured Progressive Matrixes; EF, executive functioning; GI, glycaemic index; GL, glycaemic load.

* Corresponding author: Assistant Professor J. F. W. Cohen, email cohenj@merrimack.edu 
The aim of this systematic review was to document the impact of healthier dietary consumption on EF in children and adolescents. We included studies of overall food quality (including dietary patterns), macronutrients and foods. The research included also extended to studies of other meals beyond breakfast.

\section{Methods}

\section{Literature search}

Electronic databases searched included PubMed (1975-April 2016), Education Resources Information Center (ERIC) database (1966-April 2016), PyschINFO (1967-April 2016) and Thomson Reuters' Web of Science (1900-April 2016). Inclusion criteria included studies and reviews that were related to healthier diets or food consumption compared with less-healthy diets (not fasting) and their association with $\mathrm{EF}$ in children or adolescents aged 6-18 years. This age range was selected as this is an important time period of neurogenesis related to EF, and EF skills would have typically developed sufficiently to measure by this time period ${ }^{(6)}$. Publications were not limited by geographical area to enable inclusion of both higher- and lower-income countries, and all summary measures (e.g. risk ratios, difference in means) were included. All types of studies were examined, including cross-sectional, longitudinal and experimental study designs.

The following search terms were used to search all databases (using the filters for human species and the age range 6-18 years in PubMed and PsychInfo): (nutrition OR diet OR meal pattern OR whole grain OR fruit OR vegetable OR fish) AND (executive function OR cognition OR memory OR reasoning OR attention OR learning OR problem solving OR school performance OR academic achievement OR learning/physiology OR educational measurement OR educational status OR neuropsychological tests OR inhibitory control OR impulse control). The reference lists of included manuscripts, related review articles and relevant Medical Subject Heading terms in PubMed were also examined to ensure that all publications and appropriate key words for searches were included. Abstracts were screened for relevancy, and full texts were obtained from the databases or requested from authors if they met inclusion criteria. Study screening, selection and data extraction were completed by two independent researchers in 2015 and again in 2016 (completed in April 2016), with adjudication by a third researcher when necessary. The study protocol was registered at PROSPERO International prospective register of systematic reviews (registration no. CRD42016036950).

\section{Study selection}

All study designs were included in the literature review. The definition of healthier foods was based on the recommendations from the Dietary Guidelines for Americans and Harvard's Healthy Eating Plate, which advise diets high in whole grains, fruits and vegetables, as well as lean proteins or proteins high in unsaturated fats (e.g. fish), and low in red/processed meats, SFA, trans fats and sugar ${ }^{(8,9)}$. Diets having a low glycaemic index (GI) or glycaemic load (GL) and that were consistent with these healthier guidelines or diets that were high in MUFA and PUFA were also considered healthy. 'Unhealthy' was used to describe foods or diets high in refined carbohydrates, sugar, SFA or trans fats and/or having a high GI or GL. 'Low-fat' diets that did not distinguish between healthier PUFA and MUFA and less-healthy SFA and trans fats were also considered unhealthy because these diets typically substituted the fat with highly processed, high-GI/GL foods. The cut-off values for meeting these healthier standards varied by the dietary assessment method. For feeding studies, healthy was defined as a meal with at least one of the healthier foods recommended above (e.g. whole grains, fruit, vegetable, fish, etc.) without the less-healthy items (e.g. refined grains) and/or a low-GI/GL meal. For measures of overall diet, standard scores (e.g. Healthy Eating Index (HEI) scores), nutrient substitutions (e.g. substituting SFA for PUFA) or comparisons of higher $v$. lower levels of macronutrients were used to determine whether a diet was healthy. Authors were contacted when 'healthy' was unclear. Studies were excluded if they included non-human subjects, compared food consumption with fasting only, had an unknown definition of healthy (and there was no clarification from the contacted author), had a definition of healthy greatly different from the review criteria (e.g. defined refined carbohydrates as healthy), had measures of EF combined with additional outcomes outside the scope of the review, which could not be disaggregated, or included malnourished or severely micronutrient-deficient populations (as defined by the publication). Studies that compared fasting with healthier diets were included if they also examined healthy $v$. less-healthy diets in separate analyses (results of fasting $v$. the other diets were not included in this review). Micronutrient supplementation was not examined, as this has been previously systematically evaluated ${ }^{(10,11)}$. In addition, trials of other supplements (e.g. $n$-3 supplementation) were not included in the review; this had minimal impact on the number of studies available, as studies examining cognitive functioning and supplementation have been primarily carried out among pregnant women, infants or adults or have been conducted among malnourished children or children with developmental problems ${ }^{(12)}$

Publications examining GI or GL were included as both GI and GL measure carbohydrate quality. GI is calculated by measuring the two-hour incremental area under the blood glucose curve after consuming a food portion that contains $50 \mathrm{~g}$ of carbohydrates, compared with a control of white bread or glucose $^{(13)}$. Foods such as refined carbohydrates and white potatoes/potato products typically have a high GI value $(G I \geq 70)$ because they cause rapid spikes in blood sugar levels ${ }^{(14)}$. Vegetables (excluding potatoes), fruits and legumes typically have a low GI $(\leq 55)$, whereas whole grains tend to have a moderate GI (56-69). GL, which accounts for both the impact of the carbohydrate on blood sugar levels and the amount of carbohydrate in a serving of the food, is calculated by multiplying a food's GI by the amount of carbohydrates in the food. Foods with low GL $(\leq 10)$ include fruits and vegetables that are high in fibre (excluding potatoes) and many beans/legumes such as chickpeas and black beans. Foods with a high GL $(\geq 20)$ include refined carbohydrates, white rice and 
white potatoes/potato products. Whole grains such as brown rice or oatmeal typically have medium GL.

Studies that met all these criteria and examined food quality or macronutrients are included in Table $1^{(15-26)}$, and studies that examined specific foods are included in Table $2^{(17,27-35)}$. Information extracted from each study included the following: (1) study design and duration, (2) study participant characteristics (age and country of origin), (3) types of measures for the exposures and outcomes (including whether they were validated tools) and (4) study quality (blinding of personnel, risk of attrition bias, etc.).

\section{Study quality}

All publications meeting the criteria were assessed for study quality (Table 3). Relevant components of study quality were based on the Cochrane Handbook for Systematic Reviews of Effectiveness of Interventions. Criteria for judging risk of bias in the 'Risk of bias' assessment tool were evaluated by two independent researchers $^{(36)}$. No studies were excluded on the basis of study quality if they met the other inclusion criteria. A summary score was not calculated because of concerns regarding subjectivity and a lack of consensus criteria for creating summary scores.

\section{Results}

Searches in PubMed for studies relevant to healthier diets and EF retrieved 2760 articles, whereas ERIC generated 907 articles, PsycINFO produced 569 articles and Thomson Reuters' Web of Science retrieved 1493 articles.

Of the total 4870 publications, 1650 were duplicates. Of the 3220 publications screened, 3137 were determined not relevant to the review topic based on their titles and abstracts. Among the remaining eighty-three articles, sixty-two were excluded because of a definition of healthy/unhealthy that was unclear or differed greatly from the review criteria, the grouping of EF measures with additional outcomes that were not relevant to $\mathrm{EF}$, the comparison of healthier food consumption with fasting only or the examination of malnourished populations. The remaining twenty-one articles are discussed in this review. The flow chart for the systematic review is presented in Fig. 1.

\section{Studies examining food quality or macronutrients}

Healthy dietary patterns. Among all, three studies were of overall dietary patterns and EF. Nyaradi et al. ${ }^{(23)}$ examined 'Healthy' dietary patterns (i.e. high in fruits, vegetables, whole grains, legumes and fish) $v$. 'Western' dietary patterns (i.e. high in red and processed meat, sugar-sweetened beverages, fried and refined food, and takeout foods) using FFQ, and also assessed working memory among 602 teens aged 14-17 years in Perth, Western Australia. This study found that the 'Healthy' dietary pattern was associated with fewer errors on the Groton Maze Learning Test $(\beta=-0.023 ; P=0 \cdot 035)$. Haapala et al. ${ }^{(17)}$ studied the association between non-verbal fluid intelligence/ abstract reasoning and overall diet quality among 428 children aged 6-8 years in Finland. This study calculated both Dietary Approaches to Stop Hypertension (DASH) scores and Baltic Sea
Diet Scores (BSDS) based on 4-d food records and assessed cognitive performance with the Raven's Coloured Progressive Matrixes (CPM). Overall, this study found that both lower DASH and BSDS scores were associated with lower Raven's CPM scores (i.e. poorer cognitive performance). Khan et al. examined the association between inhibitory control, fibre intake and overall dietary quality among sixty-five prepubertal children between 7 and 9 years of age by calculating a HEI score for the diets of the participants using 3-d dietary records; HEI scores are a measure of diet quality as determined by US Dietary Guidelines for Americans and are consistent with the DASH diet and Harvard's Healthy Eating Pyramid ${ }^{(19,37,38)}$. Using a modified Flanker task, which assesses cognitive inhibitory control, this study found that higher HEI scores were associated with greater performance accuracy on incongruent task trials and inversely associated with response accuracy interference, suggesting greater cognitive flexibility during increased task demands. Dietary fibre was also positively associated with task accuracy on both congruent and incongruent trials.

Glycaemic index/load. The majority of publications have been school-based feeding studies with a cross-over design. Ingwersen et al. ${ }^{(39)}$ examined the impact of a high- $v$. low-GI breakfast on attention and memory among 6-11-year-olds ( $n$ 64), and found that following consumption of a high-GI breakfast cereal children demonstrated a larger decline in performance on accuracy of attention but had better secondary memory performance, when compared with children consuming a low-GI breakfast cereal, and no association with speed of attention or memory. Benton et al. ${ }^{(15)}$ examined GL and memory among 5-7-year-olds ( $n$ 19) in a cross-over study and found that GL was inversely correlated with verbal memory $(r-0.40 ; P<0.05)$, but there was no significant association noted between GL and spatial memory, delayed verbal memory or delayed spatial memory. In another study, Micha et al. examined both GI and GL among 11-14-year-olds ( $n$ 74) using a randomised, crossover design, and in contrast to previous research they reported that a low-GI meal was associated with significantly improved performance on a word-generation task, which examined declarative verbal memory. The authors also noted that students had better scores on a Stroop task examining response time after consuming high-GI/ high-GL meals and that they performed faster on the Serial sevens test after high-GI meals compared with low-GI meals (for both low- and high-GL meals) ${ }^{(22)}$. It is of note, however, that the authors did not report whether there were significant differences in task accuracy, which is critical for interpreting the results; faster response times on a task of inhibitory control with no measure of accuracy do not necessarily suggest improved performance relative to those with slower response times, but may in fact be suggestive of an impulsive response style. Cooper et al. ${ }^{(16)}$ examined 12-14-year-olds ( $n$ 41) and reported that, although response time on the Stroop test was faster with the high-GI breakfast, improved accuracy on this test was observed following consumption of a low-GI breakfast compared with a high-GI breakfast, and response times and accuracy on the Sternberg Paradigm test and accuracy on the Flanker test improved with a low-GI breakfast. 


\begin{tabular}{|c|c|c|c|c|c|c|}
\hline References & Study design & Participants & Dietary measures & $\begin{array}{l}\text { Executive functioning } \\
\text { measure(s) }\end{array}$ & Reported findings & $\begin{array}{l}\text { Quality } \\
\text { assessment }\end{array}$ \\
\hline Benton et al. ${ }^{(15)}$ & $\begin{array}{l}\text { School-based feeding } \\
\text { study with a cross-over } \\
\text { design }\end{array}$ & $\begin{array}{l}n 19,5-7 \text {-year-old } \\
\text { children in the UK }\end{array}$ & $\begin{array}{l}\text { High- } v \text {. medium- } v \text {. low-GL } \\
\text { breakfast. Each student } \\
\text { received a high-, medium- } \\
\text { and low-GL school } \\
\text { breakfast and had } 2 \mathrm{~d} \text { of } \\
\text { testing for each meal type }\end{array}$ & $\begin{array}{l}\text { Tests based on the Recall of } \\
\text { Objects test of the British } \\
\text { Ability Scale } \\
\text { Verbal memory } \\
\text { Spatial memory } \\
\text { Delayed verbal memory } \\
\text { Delayed spatial memory }\end{array}$ & $\begin{array}{l}\text { Verbal memory: better with low-GL breakfast } \\
\text { No effects on other tests }\end{array}$ & $\mathrm{SB}, \mathrm{CE}, \mathrm{AB}, \mathrm{BP}, \mathrm{V}$ \\
\hline Cooper et al. ${ }^{(16)}$ & $\begin{array}{l}\text { School-based feeding } \\
\text { study with a cross-over } \\
\text { design }\end{array}$ & $\begin{array}{l}n 41,12-14 \text {-year-old } \\
\text { adolescents in } \\
\text { the UK }\end{array}$ & $\begin{array}{l}\text { High-GI breakfast } v \text {. } \\
\text { a low-GI breakfast } \\
\text { ( } v \text {. breakfast omission) }\end{array}$ & $\begin{array}{l}\text { Stroop test } \\
\text { Sternberg paradigm } \\
\text { Flanker task }\end{array}$ & $\begin{array}{l}\text { Stroop test: response times were faster } \\
\text { with the high-Gl breakfast } \\
\text { Overall accuracy was better and was better maintained } \\
\text { with a low-Gl breakfast } \\
\text { Sternberg paradigm: greater improvement in response } \\
\text { times and accuracy better maintained with a low-Gl } \\
\text { breakfast } \\
\text { Flanker task: accuracy better maintained } \\
\text { with a low-Gl breakfast }\end{array}$ & $\mathrm{SB}, \mathrm{CE}, \mathrm{AB}, \mathrm{V}$ \\
\hline Haapala et al. ${ }^{(17)}$ & Cross-sectional study & $\begin{array}{l}n 512,6-8 \text {-year-old } \\
\text { children in Finland }\end{array}$ & $\begin{array}{l}\text { 4-d dietary records were } \\
\text { collected to determine } \\
\text { DASH diet scores and } \\
\text { BSDS }\end{array}$ & Raven's CPM & $\begin{array}{l}\text { Lower DASH and BSDS scores were associated with } \\
\text { lower Raven's CPM scores (i.e. poorer cognitive } \\
\text { performance) }\end{array}$ & $\mathrm{V}, \mathrm{CC}$ \\
\hline $\begin{array}{l}\text { Ingwersen } \\
\text { et al. }\end{array}$ & $\begin{array}{l}\text { School-based feeding } \\
\text { study with a cross-over } \\
\text { design }\end{array}$ & $\begin{array}{l}n 64,6-11 \text {-year-old } \\
\text { children in the UK }\end{array}$ & $\begin{array}{l}\text { High-Gl breakfast (Coco } \\
\text { Pops) v. a low-Gl } \\
\text { breakfast (All-Bran) on } \\
2 \text { consecutive days }\end{array}$ & $\begin{array}{l}\text { CDR Computerized } \\
\text { Assessment Battery } \\
\text { Speed of Attention } \\
\text { Accuracy of Attention } \\
\text { Working Memory } \\
\text { Secondary Memory }\end{array}$ & $\begin{array}{l}\text { Accuracy of attention: High Gl had a larger decline in } \\
\text { performance over time } \\
\text { Secondary memory: better performance after low-GI } \\
\text { cereal compared with high-Gl cereal at both measured } \\
\text { times } \\
\text { No effects on other tests }\end{array}$ & $S B, C E, A B, V$ \\
\hline Khan et al. ${ }^{(19)}$ & Cross-sectional study & $\begin{array}{l}n 65,7-9 \text {-year-old } \\
\text { children in the USA }\end{array}$ & $\begin{array}{l}\text { 3-d food records were used } \\
\text { to assess dietary fibre, } \\
\text { total fat, SFA, } n-3 \text { fatty } \\
\text { acids, total PUFA and } \\
\text { overall diet quality (based } \\
\text { on Healthy Eating Index- } \\
2005 \text { scores) }\end{array}$ & Flanker task & $\begin{array}{l}\text { Congruent accuracy was associated with total and } \\
\text { insoluble dietary fibre } \\
\text { Incongruent accuracy was associated with total and } \\
\text { insoluble dietary fibre and diet quality score } \\
\text { Response accuracy interference was inversely associated } \\
\text { with diet quality and positively associated with greater } \\
\text { total fat consumption }\end{array}$ & $\mathrm{V}, \mathrm{CC}$ \\
\hline Khan et al. ${ }^{(20)}$ & Cross-sectional study & $\begin{array}{l}n 150,7-10 \text {-year-old } \\
\text { children in the USA }\end{array}$ & $\begin{array}{l}\text { One } 24-h \text { food recall to asses } \\
\text { carbohydrate, protein and } \\
\text { fat intake }\end{array}$ & Colour-shape switch task & $\begin{array}{l}\text { Greater total fat intake was associated with greater local } \\
\text { switch task costs for accuracy } \\
\text { Greater SFA and cholesterol intake was associated with } \\
\text { greater 'switch' costs for accuracy and reaction time }\end{array}$ & $\mathrm{V}, \mathrm{CC}$ \\
\hline Micha et al. ${ }^{(21)}$ & Observational study & $\begin{array}{l}n 60,11-14 \text {-year-old } \\
\text { children and } \\
\text { adolescents } \\
\text { in England }\end{array}$ & $\begin{array}{l}\text { Calculated the } \mathrm{Gl} \text { and } \mathrm{GL} \text { of } \\
\text { the participant's breakfast }\end{array}$ & $\begin{array}{l}\text { Serial sevens } \\
\text { Stroop task } \\
\text { Delayed word recall } \\
\text { Immediate word recall }\end{array}$ & $\begin{array}{l}\text { Serials seven: better scores with low-GI meals and } \\
\text { high-GL meals } \\
\text { Immediate word recall: better scores with high-GI meals } \\
\text { No effect on other tests }\end{array}$ & $A B, V, C C$ \\
\hline Micha et al. ${ }^{(22)}$ & $\begin{array}{l}\text { School-based feeding } \\
\text { study with students } \\
\text { randomised to GL } \\
\text { status and a cross- } \\
\text { over design for Gl }\end{array}$ & $\begin{array}{l}74,11-14 \text {-year-old } \\
\text { children and } \\
\text { adolescents in } \\
\text { England }\end{array}$ & $\begin{array}{l}\text { High-GL/high-GI v. high-GL/ } \\
\text { low-GI v. low-GL/high-GI v. } \\
\text { low-GL/low-Gl }\end{array}$ & $\begin{array}{l}\text { Serial sevens } \\
\text { Stroop task } \\
\text { Delayed word recall } \\
\text { Immediate word recall word- } \\
\text { generation task }\end{array}$ & $\begin{array}{l}\text { Serial sevens: faster performance with high-GI meals } \\
\text { (for both low- and high-GL meals) } \\
\text { Stroop task: faster performance with high-GI meals } \\
\text { (high-GL meals only) } \\
\text { Word-generation task: better scores with low-GI meals } \\
\text { (for both low- and high-GL meals) } \\
\text { No effect on other tests }\end{array}$ & SB, CE, BP, V, CC \\
\hline
\end{tabular}




\section{N British Journal of Nutrition}

Table 1. Continued

\begin{tabular}{|c|c|c|c|c|c|c|}
\hline References & Study design & Participants & Dietary measures & $\begin{array}{l}\text { Executive functioning } \\
\text { measure(s) }\end{array}$ & Reported findings & $\begin{array}{l}\text { Quality } \\
\text { assessment }\end{array}$ \\
\hline Nyaradi et al. ${ }^{(23)}$ & Longitudinal study & $\begin{array}{l}n 602,14 \text { and } \\
17 \text {-year-old } \\
\text { adolescents in } \\
\text { Australia }\end{array}$ & $\begin{array}{l}\text { Semi-quantitative FFQ was } \\
\text { used to assess 'Healthy' } \\
\text { (high in fruits, vegetables, } \\
\text { whole grains, legumes and } \\
\text { fish) and 'Western' (high in } \\
\text { red and processed meats, } \\
\text { sugar-sweetened } \\
\text { beverages, fried and } \\
\text { refined food, and take- } \\
\text { away foods) dietary } \\
\text { patterns }\end{array}$ & GML & $\begin{array}{l}\text { GML: 'Healthy' dietary pattern was associated with fewer } \\
\text { errors }\end{array}$ & $\mathrm{V}, \mathrm{CC}$ \\
\hline $\begin{array}{l}\text { Sheppard \& } \\
\quad \text { Cheatham }\end{array}$ & Cross-sectional study & $\begin{array}{l}n 70,7-9 \text {-year-old } \\
\text { children in the USA }\end{array}$ & $\begin{array}{l}\text { Three 24-h dietary recalls } \\
\text { were used to assess } n-3 \\
\text { and } n-6 \text { PUFA and the } \\
\text { ratio of } n-6: n-3\end{array}$ & $\begin{array}{l}\text { CANTAB } \\
\text { Spatial working memory } \\
\text { Spatial span } \\
\text { Stockings of Cambridge }\end{array}$ & $\begin{array}{l}\text { SWM: lower } n-6: n-3 \text { ratio was associated with greater } \\
\text { working memory } \\
\text { SSP: no effect } \\
\text { SOC: lower } n-6: n-3 \text { ratio was associated } \\
\text { with better performance on planning tasks } \\
\text { (faster times) }\end{array}$ & $\mathrm{V}, \mathrm{CC}$ \\
\hline Smith \& Foster ${ }^{(25)}$ & Randomised trial & $\begin{array}{l}n 36,14-17 \text {-year-old } \\
\text { adolescents in } \\
\text { Australia }\end{array}$ & $\begin{array}{l}\text { Low-GI breakfast (All-Bran } \\
\text { cereal and milk) v. high-GI } \\
\text { breakfast (corn flakes) }\end{array}$ & $\begin{array}{l}\text { CVLT } \\
\text { Immediate free recall } \\
\text { List A } \\
\text { Immediate free recall } \\
\text { List B } \\
\text { Short-delay free recall } \\
\text { Short-delay cued recall }\end{array}$ & No effect on recall tests & $\mathrm{SB}, \mathrm{CE}, \mathrm{AB}$ \\
\hline Zhang et al. ${ }^{(26)}$ & Cross-sectional study & $\begin{array}{l}n 3666,6-16 \text {-year- } \\
\text { old children and } \\
\text { adolescents in } \\
\text { the USA }\end{array}$ & $\begin{array}{l}\text { 24-h recalls were used to } \\
\text { assess total fat, SFA, } \\
\text { PUFA, MUFA and } \\
\text { cholesterol }\end{array}$ & $\begin{array}{l}\text { WISC-R } \\
\text { DST } \\
\text { Block-design test }\end{array}$ & $\begin{array}{l}\text { Using nutrient density models, substituting PUFA for SFA } \\
\text { or carbohydrates was associated better DST scores } \\
\text { Using nutrient residual models, there was a positive } \\
\text { association between PUFA and better DST scores and } \\
\text { an inverse association between DST scores and } \\
\text { cholesterol. } \\
\text { No effect on block design, or other nutrient density or } \\
\text { nutrient residual models }\end{array}$ & $\mathrm{SB}, \mathrm{BP}, \mathrm{V}, \mathrm{CC}$ \\
\hline
\end{tabular}

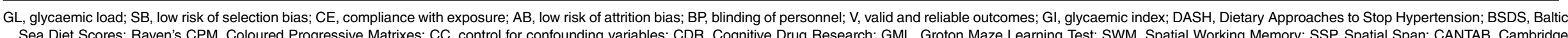
Neuropsychological Test Assessment Battery; SOC, Stockings of Cambridge; CVLT, California Verbal Learning Test; WISC-R, Wechsler Intelligence Scale for Children, Revised; DST, digit span test. 
Table 2. Studies of foods

\begin{tabular}{|c|c|c|c|c|c|c|}
\hline References & Study design & Participants & Dietary measures & EF measure(s) & Reported findings & $\begin{array}{l}\text { Quality } \\
\text { assessment }\end{array}$ \\
\hline Ames et al. ${ }^{(27)}$ & Cross-sectional study & $\begin{array}{l}n \text { 198, 14-17-year- } \\
\text { old adolescents in } \\
\text { the USA }\end{array}$ & $\begin{array}{l}\text { Semi-quantitative FFQ was } \\
\text { used to assess sugar- } \\
\text { sweetened beverage, } \\
\text { sweet snack and salty/fatty } \\
\text { snack intake }\end{array}$ & $\begin{array}{l}\text { IGT } \\
\text { Generic Go/No-Go } \\
\text { Food-cued Go/No-Go }\end{array}$ & $\begin{array}{l}\text { IGT: poorer decision making associated } \\
\text { with sugar-sweetened beverage } \\
\text { consumption among males } \\
\text { Generic Go/No-Go: inhibitory problems } \\
\text { associated with sugar-sweetened } \\
\text { beverage, sweet snack and salty/fatty } \\
\text { snack consumption among males } \\
\text { Food-cued Go/No-Go: inhibitory problems } \\
\text { with sweet-snack consumption among } \\
\text { males }\end{array}$ & $\mathrm{SB}, \mathrm{V}$ \\
\hline Chung et al. ${ }^{(28)}$ & $\begin{array}{l}9 \text { week single-blind, randomised, } \\
\text { controlled, parallel experiment }\end{array}$ & $\begin{array}{l}n 26,15-17 \text {-year-old } \\
\text { adolescents in } \\
\text { Korea }\end{array}$ & $\begin{array}{l}\text { Participants were given white } \\
\text { rice or a mixture of brown } \\
\text { rice, beans and walnuts }\end{array}$ & $\begin{array}{l}\text { A-CPT } \\
\text { DST } \\
\text { Stroop test } \\
\text { Trail making test A } \\
\text { Trail making test B } \\
\text { Wisconsin card sorting test } \\
\text { Verbal learning test } \\
\text { AX-CPT }\end{array}$ & $\begin{array}{l}\text { Verbal Learning Test (delayed recall at } \\
20 \text { min): better among the white rice } \\
\text { group at the end of } 9 \text { weeks } \\
\text { AX-CPT: white rice group had a significant } \\
\text { decrease in correct responses and } \\
\text { increase in omission errors at the end of } \\
9 \text { weeks } \\
\text { No effects on other tests }\end{array}$ & $\begin{array}{l}\text { SB, CE, AB, BP, } \\
\quad \vee, C C\end{array}$ \\
\hline Haapala et al. ${ }^{(17)}$ & Cross-sectional study & $\begin{array}{l}n 428,6-8 \text {-year-old } \\
\text { children in Finland }\end{array}$ & $\begin{array}{l}\text { 4-d dietary records were } \\
\text { collected to determine } \\
\text { fruit, vegetable, fish, } \\
\text { high-fibre grains, low-fat } \\
\text { milk, sugar-sweetened } \\
\text { beverage and red/ } \\
\text { processed meat } \\
\text { consumption }\end{array}$ & Raven's CPM & $\begin{array}{l}\text { Lower fruit and greater red/processed meat } \\
\text { consumption were associated with lower } \\
\text { Raven's CPM scores (i.e. poorer } \\
\text { cognitive performance). Lower vegetable } \\
\text { consumption was associated with lower } \\
\text { Raven's CPM scores among males only } \\
\text { Males in the lowest quartile of intake of } \\
\text { fruits, vegetables, high-fibre grains, and } \\
\text { fish, and with the highest intakes of red/ } \\
\text { processed meats had the lowest Raven's } \\
\text { CPM scores compared with males in the } \\
\text { highest quartile }\end{array}$ & $\mathrm{V}, \mathrm{CC}$ \\
\hline Mahoney et al. ${ }^{(29)}$ & $\begin{array}{l}\text { School-based feeding study with a cross- } \\
\text { over design }\end{array}$ & $\begin{array}{l}n 60,6-11 \text {-year-old } \\
\text { children in the USA }\end{array}$ & $\begin{array}{l}\text { Participants were given } \\
\text { instant oatmeal or ready- } \\
\text { to-eat, refined cereal } \\
\text { (or no breakfast). One day } \\
\text { of testing for each meal } \\
\text { type }\end{array}$ & $\begin{array}{l}\text { A-CPT } \\
\text { CPT } \\
\text { Rey Complex Figure } \\
\quad \text { Copy and Recall Test } \\
\text { DST } \\
\text { Backward } \\
\text { Forward } \\
\text { Map Task } \\
\text { Verbal Task }\end{array}$ & $\begin{array}{l}\text { DST: females performed better in the } \\
\text { backward DST with oatmeal (both 6-8- } \\
\text { year olds and } \\
\text { 9-11-year olds). No differences for males } \\
\text { or for Forward DST (females or males) } \\
\text { Map Task: better correct and blank item } \\
\text { scores with oatmeal among } 9-11 \text {-year } \\
\text { olds } \\
\text { A-CPT test: performance better after } \\
\text { oatmeal among } 6-8 \text { year olds } \\
\text { No effects on other tests }\end{array}$ & $S B, C E, A B, B P, V$ \\
\hline Riggs et al. ${ }^{(30)}$ & Cross-sectional study & $\begin{array}{l}n \text { 107, 9-10-year-old } \\
\text { children in the USA }\end{array}$ & $\begin{array}{l}\text { Self-reported fruit, vegetable } \\
\text { and snack intakes } \\
\text { (adapted from validated } \\
\text { FFQ) }\end{array}$ & $\begin{array}{l}\text { Subscales of the BRIEF-SR } \\
\text { Inhibitory control } \\
\text { Working memory }\end{array}$ & $\begin{array}{l}\text { Inverse association between EF and snack } \\
\text { food intake } \\
\text { No association between EF and fruit or } \\
\text { vegetable intake }\end{array}$ & $\mathrm{V}, \mathrm{CC}$ \\
\hline Riggs et al. ${ }^{(31)}$ & $\begin{array}{l}\text { After-school-based study with students } \\
\text { randomised to intervention or control } \\
\text { group }\end{array}$ & $\begin{array}{l}n \text { 184, 9-10-year-old } \\
\text { children in the USA }\end{array}$ & $\begin{array}{l}\text { Self-reported fruit, vegetable, } \\
\text { and snack intakes before } \\
\text { and after intervention } \\
\text { (adapted from validated } \\
\text { FFQ) }\end{array}$ & $\begin{array}{l}\text { Subscales of the BRIEF-SR } \\
\text { Inhibitory control } \\
\text { Working memory }\end{array}$ & $\begin{array}{l}\text { Inverse association between executive } \\
\text { function and snack food intake at } \\
\text { baseline } \\
\text { Positive association between executive } \\
\text { function and fruit/vegetable intake at } \\
\text { baseline and at } 4 \text { months post- } \\
\text { intervention }\end{array}$ & $\mathrm{SB}, \mathrm{V}$ \\
\hline
\end{tabular}




\section{N British Journal of Nutrition}

Table 2. Continued

\begin{tabular}{|c|c|c|c|c|c|c|}
\hline References & Study design & Participants & Dietary measures & EF measure(s) & Reported findings & $\begin{array}{l}\text { Quality } \\
\text { assessment }\end{array}$ \\
\hline Riggs et al. ${ }^{(32)}$ & Cross-sectional study & $\begin{array}{l}n 1587,9-10 \text {-year- } \\
\text { olds in the USA }\end{array}$ & $\begin{array}{l}\text { Self-reported fruit, vegetable } \\
\text { and snack intakes } \\
\text { (adapted from validated } \\
\text { FFQ) }\end{array}$ & $\begin{array}{l}\text { Subscales of the BRIEF-SR } \\
\text { Inhibitory control } \\
\text { Working memory }\end{array}$ & $\begin{array}{l}\text { Inverse association between executive } \\
\text { function and snack food intake } \\
\text { Positive association between executive } \\
\text { function and fruit/vegetable intake }\end{array}$ & $\mathrm{V}, \mathrm{CC}$ \\
\hline Sorensen et al. ${ }^{(33)}$ & $\begin{array}{l}\text { School-based feeding study with a cross- } \\
\text { over design }\end{array}$ & $\begin{array}{l}n \text { 726, 8-11-year-old } \\
\text { children in } \\
\text { Denmark }\end{array}$ & $\begin{array}{l}\text { Fish consumption was } \\
\text { assessed using } \\
\text { biomarkers for } n \text {-3 PUFA } \\
\text { (EPA and DHA) }\end{array}$ & D2 test of attention & $\begin{array}{l}\text { Baseline PUFA levels positively associated } \\
\text { with concentration performance and } \\
\text { processing speed Impulsivity and } \\
\text { inattention were inversely correlated with } \\
\text { EPA/DHA level in males, whereas } \\
\text { inattention errors were positively } \\
\text { correlated with EPA/DHA levels in } \\
\text { females } \\
\text { Increases in EPA/DHA from the intervention } \\
\text { were associated with improved } \\
\text { performance on measures of EF }\end{array}$ & $\mathrm{SB}, \mathrm{AB}, \mathrm{V}, \mathrm{CC}$ \\
\hline $\begin{array}{l}\text { van der Wurff } \\
\quad \text { et al. }\end{array}$ & Cross-sectional study & $\begin{array}{l}n 266,13-15 \text {-year- } \\
\text { old adolescents in } \\
\text { the Netherlands }\end{array}$ & $\begin{array}{l}\text { Fish consumption } \\
\text { measured by an } \\
\text { omega-3 index } \\
\text { (EPA and DHA in } \\
\text { blood in erythrocytes } \\
\text { as a per cent of total } \\
\text { fatty acids) }\end{array}$ & $\begin{array}{l}\text { CST } \\
\text { D2 test of attention } \\
\text { DST } \\
\quad \text { Backward } \\
\quad \text { Forward } \\
\text { LDST } \\
\text { Stroop }\end{array}$ & $\begin{array}{l}\text { There was a positive association between } \\
\text { omega- } 3 \text { index and performance on the } \\
\text { LDST and D2 test of attention } \\
\text { No effect on other tests }\end{array}$ & $\mathrm{BP}, \mathrm{V}, \mathrm{CC}$ \\
\hline Wesnes et al. ${ }^{(35)}$ & Randomised, cross-over study & $\begin{array}{l}\text { 29, 9-16-year-old } \\
\text { children and } \\
\text { adolescents in } \\
\text { the UK }\end{array}$ & $\begin{array}{l}\text { Participants were given } \\
\text { Cheerios cereal, } \\
\text { Shreddies cereals or } \\
\text { glucose drink } \\
\text { (or no breakfast) }\end{array}$ & $\begin{array}{l}\text { CDR Computerised } \\
\text { Assessment Battery } \\
\text { Attention } \\
\text { Working memory } \\
\text { Episodic secondary } \\
\text { memory }\end{array}$ & $\begin{array}{l}\text { The glucose drink was associated with } \\
\text { greater declines in attention and episodic } \\
\text { secondary memory compared with the } \\
\text { two cereal conditions } \\
\text { No effect on working memory }\end{array}$ & SB, CE, AB \\
\hline
\end{tabular}

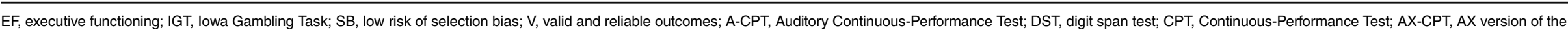

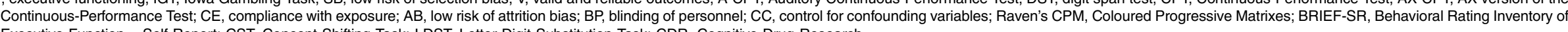
Executive Function - Self-Report; CST, Concept Shifting Task; LDST, Letter Digit Substitution Task; CDR, Cognitive Drug Research. 
Table 3. Quality assessment of studies

\begin{tabular}{|c|c|c|c|c|c|c|}
\hline References & $\begin{array}{l}\text { Low risk of } \\
\text { selection bias }\end{array}$ & $\begin{array}{c}\text { Compliance with } \\
\text { exposure }\end{array}$ & $\begin{array}{l}\text { Low risk of } \\
\text { attrition bias }\end{array}$ & Blinding of personnel & $\begin{array}{c}\text { Valid and reliable outcomes, } \\
\text { for example appropriateness } \\
\text { of cognitive test }\end{array}$ & Control for confounding variables \\
\hline Ames et al. ${ }^{(27)}$ & + & $\mathrm{N} / \mathrm{A}$ & $\mathrm{N} / \mathrm{A}$ & Not enough information given & + & Examined sex separately \\
\hline Benton et al. ${ }^{(15)}$ & + & + & + & + & + & $\begin{array}{l}\text { Sex (not included in models as there were no } \\
\text { differences by sex) }\end{array}$ \\
\hline Chung et al. ${ }^{(28)}$ & + & + & + & + & + & $\begin{array}{l}\text { Randomised by age, education, height, weight and } \\
\text { BMI }\end{array}$ \\
\hline Cooper et al. ${ }^{(16)}$ & + & + & + & Not enough information given & + & \\
\hline Haapala et al. ${ }^{(17)}$ & & N/A & N/A & Not enough information given & + & $\begin{array}{l}\text { Age, sex, parental education, and household } \\
\text { income, total physical activity, total screen-based } \\
\text { sedentary behaviour, cardiovascular } \\
\text { performance, motor performance, body fat } \\
\text { percentage, clinical puberty, current height as a } \\
\text { percentage of the predicted adult height, } \\
\text { developmental disorders, skipping meals, eating } \\
\text { behaviours, and total energy }\end{array}$ \\
\hline Ingwersen et al. ${ }^{(39)}$ & + & + & + & Not enough information given & + & Examined sex separately \\
\hline Khan et al. ${ }^{(19)}$ & & N/A & N/A & Not enough information given & + & $\begin{array}{l}\text { Age, pubertal stage, intelligence quotient, } \mathrm{VO}_{2 \max } \\
\text { and per cent fat mass (sex and SES were not } \\
\text { significant confounders) }\end{array}$ \\
\hline Khan et al. ${ }^{(20)}$ & & $\mathrm{N} / \mathrm{A}$ & $\mathrm{N} / \mathrm{A}$ & Not enough information given & + & $\begin{array}{l}\text { Age, sex, SES, Kaufman Brief Intelligence Test, } \\
\mathrm{VO}_{2 \max }, \mathrm{BMI} \text {, and total energy intake }\end{array}$ \\
\hline Mahoney et al.(29) & + & + & + & + & + & \\
\hline Micha et al. ${ }^{(21)}$ & + & $\mathrm{N} / \mathrm{A}$ & + & Not enough information given & + & $\begin{array}{l}\text { Sex, SES, age, height, weight, BMI, Hb levels, } \\
\text { blood glucose levels, 'happy' mood score before } \\
\text { the cognitive function tests, and time between } \\
\text { breakfast and the first cognitive function test }\end{array}$ \\
\hline Micha et al. ${ }^{(22)}$ & + & + & & + & + & $\begin{array}{l}\text { Order of administration of breakfast meals, sex, } \\
\text { age, height, weight, BMl, and glucose, and } \\
\text { cortisol levels at baseline }\end{array}$ \\
\hline Nyaradi et al. ${ }^{(23)}$ & & & & Not enough information given & + & $\begin{array}{l}\text { Maternal education, family income, the presence of } \\
\text { biological father in the family, family functioning } \\
\text { and sex }\end{array}$ \\
\hline Riggs et al. ${ }^{(30)}$ & & $\mathrm{N} / \mathrm{A}$ & $\mathrm{N} / \mathrm{A}$ & $\mathrm{N} / \mathrm{A}$ & + & Sex, ethnicity, SES and academic achievement \\
\hline Riggs et al. ${ }^{(31)}$ & + & $\begin{array}{l}\text { Not enough } \\
\text { information given }\end{array}$ & & Not enough information given & + & \\
\hline Riggs et al. ${ }^{(32)}$ & $\begin{array}{l}\text { Not enough } \\
\text { information given }\end{array}$ & N/A & N/A & $\mathrm{N} / \mathrm{A}$ & + & $\begin{array}{l}\text { Age, sex, ethnicity, SES (student free lunch status) } \\
\text { and school grades }\end{array}$ \\
\hline Sheppard \& Cheatham ${ }^{(24)}$ & & N/A & N/A & Not enough information given & + & $\begin{array}{l}\text { Maternal education, physical activity, total energy } \\
\text { content, data collection time period (paternal } \\
\text { education, sex and breast-fed status as an infant } \\
\text { were not significant confounders) }\end{array}$ \\
\hline Smith \& Foster (25) & + & + & + & Not enough information given & & \\
\hline Sorensen et al. ${ }^{(33)}$ & + & & + & & + & $\begin{array}{l}\text { Individual (as a random effect to account for } \\
\text { repeated measures), baseline value of the } \\
\text { cognitive test, intervention order, sex, household } \\
\text { intervention, year group, baseline age, and } \\
\text { month of baseline test }\end{array}$ \\
\hline van der Wurff et al. ${ }^{(34)}$ & & $\mathrm{N} / \mathrm{A}$ & $\mathrm{N} / \mathrm{A}$ & + & + & $\begin{array}{l}\text { BMI, sex, age, alcohol consumption, smoking } \\
\text { status, parental education }\end{array}$ \\
\hline $\begin{array}{l}\text { Wesnes et al.(35) } \\
\text { Zhang et al. }{ }^{(26)}\end{array}$ & $\begin{array}{l}+ \\
+\end{array}$ & $\stackrel{+}{N / A}$ & $\stackrel{+}{N / A}$ & $\begin{array}{c}\text { Not enough information given } \\
+\end{array}$ & + & $\begin{array}{l}\text { Ethnicity, maternal education, rural/urban } \\
\text { classification of residence area, maternal marital } \\
\text { status, poverty status, food insufficient, stature- } \\
\text { for-age } Z \text {-score, BMI-for-age, Fe deficiency, } \\
\text { health status, substance abuse, physical activity }\end{array}$ \\
\hline
\end{tabular}

SES, socio-economic status. 


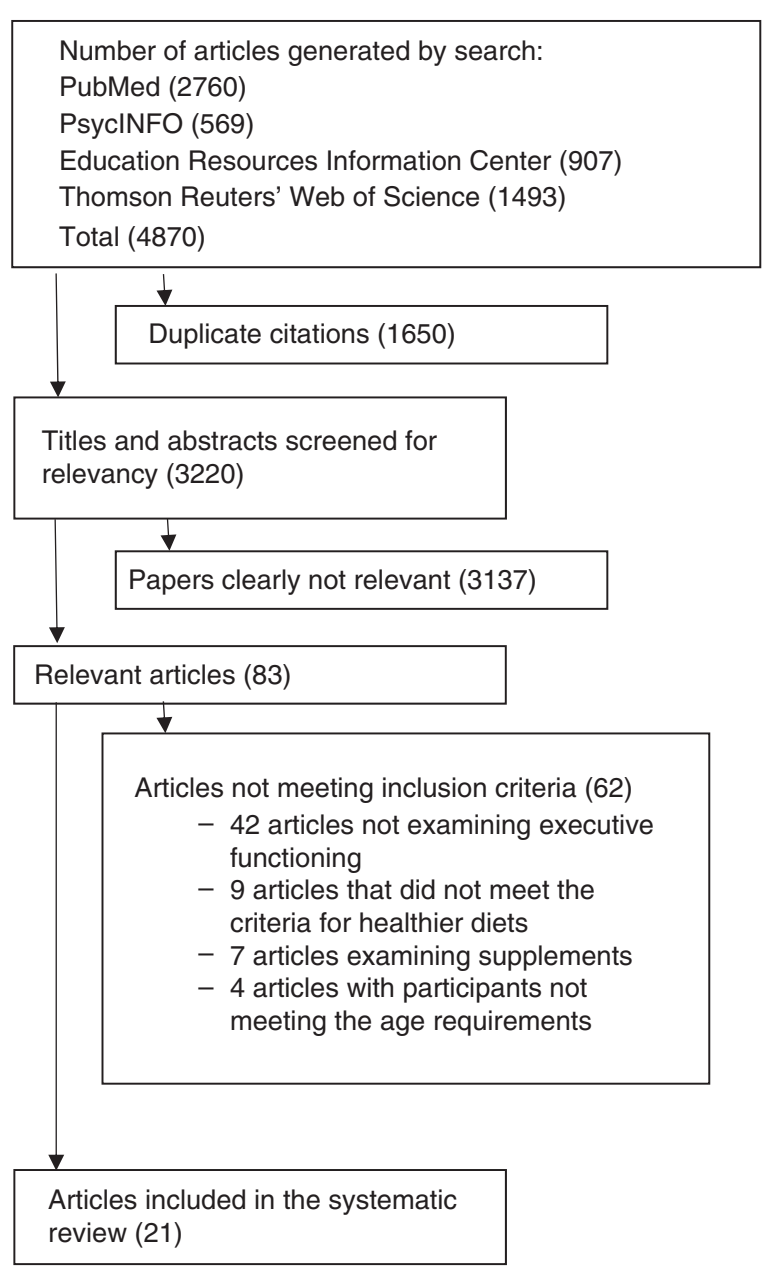

Fig. 1. Flow chart showing the number of articles at each stage of the search.

Among the studies that did not use a cross-over design, one study conducted a randomised trial among Australian 14-17-year-olds ( $n$ 36) using the Modified California Verbal Learning Test and found no association between GI and immediate or short-delay free recall ${ }^{(25)}$. An observational study among 11-14-year-olds ( $n$ 60) found that students had better immediate word recall task scores with high-GI meals compared with low-GI meals (for both high- and low-GL meals), but no effect of GI on delayed word recall ${ }^{(21)}$.

Macronutrients. Four cross-sectional studies included in the present systematic review examined differences in macronutrients. In a study that used data from 3666 children and adolescents participating in the Third National Health and Nutrition Examination Survey to examine macronutrient distributions, each 5\% increase in energy from PUFA was associated with a decreased odds of poor performance in the digit span test (working memory) when replacing SFA (OR 0.58; CI $0.37,0.91$ ) or carbohydrates (OR 0.61; CI $0.43,0 \cdot 88)^{(26)}$. In nutrient residual models, greater consumption of PUFA was associated with deceased odds of poor digit span scores (OR 0.74; CI 0.58, 0.94), and high cholesterol intake was associated with increased odds of poor digit span scores (OR 1.25; CI 1.11, 1.42). No significant associations were seen between macronutrients and a measure of spatial ability, the Block design test. In addition, a study conducted by Khan et $a l^{(19)}$ examining 7-9-year-olds ( $n$ 65) found that greater overall fat consumption was associated with higher task interference, suggesting poorer maintenance of cognitive control, but reported no association between SFA, $n-3$ fatty acids or PUFA and EF. A second study by Khan et al. ${ }^{(20)}$ that used a taskswitching paradigm to measure cognitive flexibility examined carbohydrate, protein and fat intake among 7-10-year-olds ( $n$ 150). This study also found that higher total fat intake was associated with longer reaction times during the task condition, which required greater cognitive flexibility. Further, the authors reported a correlation between greater SFA and cholesterol intakes and higher 'switch' costs, which represent difficulty in maintaining multiple cognitive sets in working memory and are indicative of less-efficient executive control processes. Finally, a cross-sectional study among 7-9-year-old children ( $n$ 70), conducted by Sheppard \& Cheatham ${ }^{(24)}$, assessed EF using measures of planning (Stockings of Cambridge (SOC)) and working memory (Spatial Working Memory and Spatial Span) from the Cambridge Neuropsychological Test Assessment Battery and assessed diet using three $24-\mathrm{h}$ recalls to determine $n-3$ and $n-6$ fatty acid levels and the ratio of $n-6: n-3$. This study found that children with lower $n-6: n-3$ ratios (i.e. consumed relatively higher levels of $n-3$ fatty acids) showed greater performance on EF tasks. In particular, they had shorter initial processing times during the spatial working memory task and had reduced average times during the planning task (SOC). In addition, a significant interaction between the overall fatty acid intake levels and their ratio was observed, with higher average $n$-3 fatty acid levels associated with greater mean planning times (i.e. poorer EF performance), in the presence of higher $n-6$ fatty acid intake (i.e. overall high quantities of the fatty acids, but a low $n-6: n-3$ ratio).

\section{Studies examining foods}

Whole grains. Several studies have examined whole grains because of their healthier nutrient profile and generally lower GI/GL values. The first was a small, randomised, cross-over study among twenty-nine children and adolescents aged 9-16 years, which found that a glucose drink was associated with significantly larger declines in attention and working memory compared with wholegrain cereal ${ }^{(35)}$. Mahoney et al. ${ }^{(29)}$ conducted a school-based, cross-over feeding study, and found that after consuming oatmeal, females performed better on the backwards digit span test, 9-11-year-old girls and boys had better scores on the Map Task, and 6-8-year olds performed better on the Auditory Continuous-Performance Test (A-CPT) compared with their performance after consuming refined breakfast cereal. The study by Haapala et al. ${ }^{(17)}$ also found that males who were in the lowest quartile of high-fibre grain consumption had the lowest scores on the Raven's CPM (non-verbal reasoning) compared with those in the highest quartile. Finally, Chung et al. ${ }^{(28)}$ implemented a randomised, controlled feeding study among 15-17-year-olds ( $n$ 26), and 
found that those who had been assigned to consume white rice had significantly greater improvements in scores on a verbal learning test after a delayed recall at $20 \mathrm{~min}$ compared with those consuming the healthier diet composed of whole grains, kidney beans and walnuts $(P<0.05)$, but not on the AX version of the Continuous-Performance Test (AX-CPT) - a measure of attention, vigilance and response impulsivity. Those consuming white rice had a significant decrease in correct responses on the AX-CPT as well as an increase in omission errors at the end of the study compared with those consuming the healthier diet ${ }^{(28)}$. There were no significant differences between the groups on the other tests administered, which included the A-CPT, digit span test, Stroop test, Trail making test A and B and the Wisconsin Card Sorting Test.

\section{Sweet and salty snacks and sugar-sweetened beverages.} Four studies examined salty and sugary snacks and beverages and found consistent associations between 'junk' foods and poorer EF. Ames et al. ${ }^{(27)}$ found that among male adolescents, greater sugar-sweetened beverage consumption was associated with poorer decision making on the Iowa Gambling Task and reduced ability to inhibit inappropriate responses on the standard Go/No-Go task. In addition, sweet and salty/fatty snacks were associated with inhibitory problems on the standard Go/No-Go task, and sweet-snack consumption was associated with inhibitory problems on the food-based Go/No-Go task among males. Multiple studies by Riggs et al. $^{(30-32)}$ that examined snack food consumption among children also found an inverse association with self-reported measures of EF.

Fruits and vegetables. Four studies examined fruits, vegetables and $\mathrm{EF}$, with three finding higher fruit and vegetable intakes associated with greater EF. In the small, cross-sectional study among 9-10-year-olds conducted by Riggs et al. $^{(30)}$, no association between fruit or vegetable consumption and $\mathrm{EF}$ was observed, which the authors noted may have been due to the small sample size. However, in the two larger studies conducted by Riggs et al. ${ }^{(31,32)}$, fruit and vegetable intakes were positively associated with EF. Finally, Haapala et al. found a positive association between fruit consumption and abstract reasoning measured using Raven's CPM. This study also found that males who were in the lowest quartile of vegetable consumption also had the lowest Raven's CPM scores compared with those in the highest quartile ${ }^{(17)}$.

Proteins. Three studies examined the consumption of animalbased sources of protein (i.e. fish or red/processed meat) and EF. Haapala et al. ${ }^{(17)}$ found that males who were in the lowest quartile of fish consumption had the lowest abstract reasoning scores. In addition, this study found that, among both males and females, greater red and processed meat consumption was associated with lower scores on the Raven's CPM. A study conducted by Sorensen et al. ${ }^{(33)}$ assessed 726 children aged 8-11 years using a school-based feeding intervention that provided students with fish twice per week. Diet was assessed using $n-3$ fatty acid biomarkers, whereas EF was measured with the D2 test of attention. The authors noted that at baseline EPA and DHA status was positively correlated with concentration performance and processing speed; in addition, impulsivity and inattention were inversely correlated with EPA/DHA level (i.e. higher EPA/DHA was associated with lower impulsivity/ inattention errors) in boys, whereas inattention errors were positively correlated with EPA/DHA levels in girls. Following the 3-month school meal intervention, EPA/DHA levels increased and were associated with improved performance on measures of EF. Finally, a study by van der Wurff et al. ${ }^{(34)}$ examined multiple domains of EF and their association with fish consumption, measured using $n$ - 3 fatty acid biomarkers to calculate an omega3 index (calculated as EPA and DHA in blood erythrocytes as a per cent of total fatty acids). Among the 266 adolescents participating in the study, there was a positive association between the omega-3 index and performance on the Letter Digit Substitution Task and fewer errors of omission on the D2 test of attention, indicating better information processing speed and less impulsivity with greater fish consumption.

\section{Discussion}

Overall, studies examining the longer-term effect of a healthier diet found consistently positive associations with EF, whereas studies examining the acute association between GI and GL and EF found mixed results, although the majority was suggestive of improved EF with lower GI/GL foods. Similarly, studies examining macronutrients were inconsistent, and the limited number of studies in this area made it difficult to draw conclusions. Studies examining specific foods primarily found that healthier foods (e.g. whole grains, fruits and/or vegetables) were positively associated with EF, whereas snack foods - which are typically high in sugar, refined grains and SFA - sugarsweetened beverages and red/processed meats were inversely associated with EF. Studies examining fish intake were also suggestive of a positive association with $\mathrm{EF}$.

Studies examining healthier diets and EF have used varying tests of cognition, which may in part help explain the inconsistent findings among those examining GI and GL. It is important to note that different versions of a cognitive task (i.e. the Stroop Color Word Task) are likely to yield different results, making comparisons and interpretation of data across studies difficult. In addition, because EF covers multiple processes (e.g. planning, working memory, inhibitory control), there is heterogeneity among the areas assessed by each of the validated tests available. Although there are a number of 'gold standard' measures of EF (i.e. the Wisconsin Card Sorting Test), measures must be tailored to fit specific population requirements, including age, education, intelligent quotient, time requirements to administer the tests and the cognitive domain of interest, which may also impact the interpretation of the results. A recent review of GI and more general cognition in both children and adults also found that the findings were inconclusive, and noted that this may also be due to the use of cognitive tests that may not be sensitive enough to detect small but meaningful variations in cognition ${ }^{(40)}$. Given the number of well-validated tests, it is therefore essential to select both appropriately sensitive tests and to assess a sufficient number of participants to ensure the power to detect changes in EF. 
It is possible that healthier foods improved EF through multiple mechanisms. Certain vitamins (e.g. B vitamins and vitamin $\mathrm{C}$ ) and minerals (e.g. Zn) are cofactors for enzymes that synthesise neurotransmitters, thus impacting cognition throughout childhood and adolescence ${ }^{(41-43)}$. In addition, micronutrients (e.g. folate) and healthy fats, particularly PUFA, impact myelin sheath integrity and nerve cell membranes, which affect neuron function ${ }^{(42)}$. Brain glucose levels may also impact $\mathrm{EF}^{(42)}$. A healthier diet may lead to changes in the brain structure and function, particularly in the frontal cortical regions involved, through synapse formation, in neurogenesis, myelination and glucose control.

Improvements in EF resulting from healthier diets can have important academic, public health and policy implications, as EF is associated with both academic performance and healthrelated decision making. As the majority of youth in the USA fail to meet dietary recommendations, policies that improve diet, and thus EF, may have important implications ${ }^{(44)}$. Schools may consider policies that further improve the school food environment, including both school meals and competitive foods, to potentially impact the academic performance and health of students. Similarly, policies that improve the content of the foods available through the Supplemental Nutrition Assistance Program (SNAP) may have important implications for children, as roughly half of SNAP participants are children aged 18 years or younger ${ }^{(45)}$

This review is subject to a number of limitations. Publication bias is a possibility, as studies of diet and EF with null findings may have been less likely to be published. Further, substantial differences emerged in the types of foods and diets examined as well as the metrics used to assess both diet and EF. This may have impacted the interpretation of the relationship between a healthier diet and EF. Finally, in many studies, it was not possible to determine the causal nature of the relationship due to the cross-sectional study designs; it is possible that, given better EF, children are able to make healthier food choices. Future, longitudinal studies examining the impact of a healthier diet on EF are warranted.

In summary, although the association between GI/GL and EF remains unclear, there appears to be a positive association between EF and healthier overall diets and foods. In addition, studies suggest that consumption of less-healthy snack foods, sugar-sweetened beverages and red/processed meats is associated with poorer EF. Overall, future short- and long-term studies should examine healthier food consumption, including whole grains, fruits, vegetables and fish in a controlled environment, such as a cross-over feeding study. Studies examining macronutrients are also warranted, and additional studies examining sugar-sweetened beverages or red/processed meats and their potential impact on EF are also indicated. The use of sensitive, validated cognitive measures is essential to determine potential associations between food and EF.

\section{Acknowledgements}

The authors thank Sara Rimm-Kaufman for her thoughtful assistance with the interpretation of the studies reviewed.

This research received no specific grant from any funding agency or from commercial or not-for-profit sectors.
The authors' contributions were as follows: J. F. W. C., E. B. R. and S. A. G. defined the scope of the project. J. F. W. C. and M. T. G. performed the literature search, reviewed identified publications and assessed study quality. J. F. W. C. wrote the manuscript with significant contributions from E. B. R., L. B. F. K., M. T. G. and S. A. G. All the authors contributed to the interpretation of the data, approved the final manuscript and took full responsibility for the final content.

None of the authors has any conflicts of interest to declare.

\section{References}

1. Blair C (2002) School readiness: integrating cognition and emotion in a neurobiological conceptualization of children's functioning at school entry. Am Psychol 57, 111-127.

2. Bierman K, Nix R, Greenberg M, et al. (2008) Executive functions and school readiness intervention: impact, moderation, and mediation in the Head Start REDI program. Dev Psychopathol 20, 821-843.

3. White H, Xie M, Thompson W, et al. (2001) Psychopathology as a predictor of adolescent drug use trajectories. Psychol Addict Behav 15, 210-218.

4. Braet C, Claus L, Verbeken S, et al. (2007) Impulsivity in overweight children. Eur Child Adolesc Psychiatry 16, 473-483.

5. Nederkoorn C, Braet C, Van Eijs Y, et al. (2006) Why obese children cannot resist food: the role of impulsivity. Eat Behav 7, 315-322.

6. Weintraub S, Dikmen SS, Heaton RK, et al. (2013) Cognition assessment using the NIH Toolbox. Neurology 80, Suppl. 3, S54-S64.

7. Hoyland A, Dye L \& Lawton C (2009) A systematic review of the effect of breakfast on the cognitive performance of children and adolescents. Nutr Res Rev 22, 220-243.

8. United States Department of Agriculture (2010) Dietary guidelines for Americans. http://www.cnpp.usda.gov/Dietary Guidelines.htm (accessed January 2015).

9. Harvard Health Publications (2011) Harvard Healthy Eating Plate. http://www.health.harvard.edu/healthy-eating-plate (accessed January 2015)

10. Eilander A, Gera T, Sachdev HS, et al. (2010) Multiple micronutrient supplementation for improving cognitive performance in children: systematic review of randomized controlled trials. Am J Clin Nutr 91, 115-130.

11. Warthon-Medina M, Moran V, Stammers A, et al. (2015) Zinc intake, status and indices of cognitive function in adults and children: a systematic review and meta-analysis. Eur J Clin Nutr 69, 649-661.

12. Kirby A, Woodward A \& Jackson S (2010) Benefits of omega-3 supplementation for shoolchildren: review of the current evidence. Br Educ Res J 36, 699-732.

13. Ludwig DS (2007) Clinical update: the low-glycaemicindex diet. Lancet 369, 890-892.

14. Foster-Powell K, Holt SH \& Brand-Miller JC (2002) International table of glycemic index and glycemic load values: 2002. Am J Clin Nutr 76, 5-56.

15. Benton D, Maconie A \& Williams C (2007) The influence of the glycaemic load of breakfast on the behaviour of children in school. Physiol Behav 92, 717-724.

16. Cooper SB, Bandelow S, Nute ML, et al. (2012) Breakfast glycaemic index and cognitive function in adolescent school children. Br J Nutr 107, 1823-1832.

17. Haapala EA, Eloranta A, Venäläinen T, et al. (2015) Associations of diet quality with cognition in children - the Physical Activity and Nutrition in Children Study. Br J Nutr 114, 1080-1087. 
18. Ingwersen J, Defeyter MA, Kennedy DO, et al. (2007) A low glycaemic index breakfast cereal preferentially prevents children's cognitive performance from declining throughout the morning. Appetite 49, 240-244.

19. Khan NA, Raine LB, Drollette ES, et al. (2015) Dietary fiber is positively associated with cognitive control among prepubertal children. J Nutr 145, 143-149.

20. Khan NA, Raine LB, Drollette ES, et al. (2015) The relation of saturated fats and dietary cholesterol to childhood cognitive flexibility. Appetite 93, 51-56.

21. Micha R, Rogers PJ \& Nelson M (2010) The glycaemic potency of breakfast and cognitive function in school children. Eur J Clin Nutr 64, 948-957.

22. Micha R, Rogers PJ \& Nelson M (2011) Glycaemic index and glycaemic load of breakfast predict cognitive function and mood in school children: a randomised controlled trial. $\mathrm{Br} \mathrm{J}$ Nutr 106, 1552-1561.

23. Nyaradi A, Foster JK, Hickling S, et al. (2014) Prospective associations between dietary patterns and cognitive performance during adolescence. J Child Psychol Psychiatry 55, 1017-1024.

24. Sheppard KW \& Cheatham CL (2013) Omega-6 to omega-3 fatty acid ratio and higher-order cognitive functions in 7-to 9-y-olds: a cross-sectional study. Am J Clin Nutr 98, 659-667.

25. Smith MA \& Foster JK (2008) The impact of a high versus a low glycaemic index breakfast cereal meal on verbal episodic memory in healthy adolescents. Nutr Neurosci 11, 219-227.

26. Zhang J, Hebert JR \& Muldoon MF (2005) Dietary fat intake is associated with psychosocial and cognitive functioning of school-aged children in the United States. J Nutr 135, 1967-1973.

27. Ames SL, Kisbu-Sakarya Y, Reynolds KD, et al. (2014) Inhibitory control effects in adolescent binge eating and consumption of sugar-sweetened beverages and snacks. Appetite 81, 180-192.

28. Chung YC, Park CH, Kwon HK, et al. (2012) Improved cognitive performance following supplementation with a mixed-grain diet in high school students: a randomized controlled trial. Nutrition 28, 165-172.

29. Mahoney CR, Taylor HA, Kanarek RB, et al. (2005) Effect of breakfast composition on cognitive processes in elementary school children. Physiol Behav 85, 635-645.

30. Riggs NR, Spruijt-Metz D, Sakuma KL, et al. (2010) Executive cognitive function and food intake in children. $J$ Nutr Educ Behav 42, 398-403.

31. Riggs N, Chou CP, Spruijt-Metz D, et al. (2010) Executive cognitive function as a correlate and predictor of child food intake and physical activity. Child Neuropsychol 16, 279-292.

32. Riggs NR, Spruijt-Metz D, Chou CP, et al. (2012) Relationships between executive cognitive function and lifetime substance use and obesity-related behaviors in fourth grade youth. Child Neuropsychol 18, 1-11.

33. Sørensen LB, Damsgaard CT, Dalskov S, et al. (2015) Diet-induced changes in iron and $n-3$ fatty acid status and associations with cognitive performance in 8-11-year-old Danish children: secondary analyses of the Optimal Well-Being, Development and Health for Danish Children through a Healthy New Nordic Diet School Meal Study. Br J Nutr 114, 1623-1637.

34. van der Wurff ISM, von Schacky C, Berge K, et al. (2016) Association between blood omega-3 index and cognition in typically developing Dutch adolescents. Nutrients $\mathbf{8}, 13$.

35. Wesnes KA, Pincock C, Richardson D, et al. (2003) Breakfast reduces declines in attention and memory over the morning in schoolchildren. Appetite 41, 329-331.

36. Higgins JPT, Altman DG \& Sterne JAC (2011) Chapter 8: assessing risk of bias in included studies. In Cochrane Handbook for Systematic Reviews of Interventions [JPT Higgins and S Green, editors]. http://handbook.cochrane. org/chapter_8/8_5_the_cochrane_collaborations_tool_for_ assessing_risk_of_bias.htm

37. Guenther PM, Reedy J \& Krebs-Smith SM (2008) Development of the healthy eating index-2005. J Am Diet Assoc 108, 1896-1901.

38. Guenther PM, Reedy J, Krebs-Smith SM, et al. (2008) Evaluation of the healthy eating index-2005. J Am Diet Assoc 108, 1854-1864

39. Ingwersen J, Defeyter MA, Kennedy DO, et al. (2007) A low glycaemic index breakfast cereal preferentially prevents children's cognitive performance from declining throughout the morning. Appetite 49, 240-244.

40. Philippou E \& Constantinou M (2014) The influence of glycemic index on cognitive functioning: a systematic review of the evidence. Adv Nutr 5, 119-130.

41. Haller J (2005) 13 Vitamins and brain function. In Nutritional Neuroscience, pp. 207-230 [HR Lieberman, RB Kanarek and C Prasad, editors]. Boca Raton, FL: CRC Press.

42. Drake V (2014) Micronutrients and cognitive function. Linus Pauling Institute Micronutrient Information Center. Corvallis, OR. http://lpi.oregonstate.edu/infocenter/cognition. html (accessed January 2015).

43. Greenwood CE \& Craig REA (1987) Dietary influences on brain function: implications during periods of neuronal maturation. Curr Topics Nutr Dis 16, 159-216.

44. Krebs-Smith SM, Guenther PM, Subar AF, et al. (2010) Americans do not meet federal dietary recommendations. J Nutr 140, 1832-1838.

45. US Department of Agriculture (2014) Supplemental Nutrition Assistance Program participation rates: fiscal years 2010 and 2011. http://www.fns.usda.gov/sites/default/files/trends20102011.pdf (accessed January 2015). 